\title{
Desempenho do Método dos Elementos de Contorno com Integração Direta em Problemas de Autovalor com Domínio Não Regular
}

\author{
Carlos Friedrich Loeffler ${ }^{1}$ \\ Programa de Pós Graduação em Engenharia Mecânica, PPGEM/UFES, Vitória, ES \\ Hercules de Melo Barcelos ${ }^{2}$ \\ Programa de Pós Graduação em Engenharia Mecânica, PPGEM/UFES, Vitória, ES
}

\begin{abstract}
Resumo. A pesquisa como um todo tem como objetivo testar os principais tipos de funções de base radial junto à nova técnica de solução do Método dos Elementos de Contorno, denominada MECID, na solução de problemas de autovalor em que o domínio bidimensional não é regular. Diversas funções radiais, tanto as funções radiais clássicas quanto as funções radiais de Wendland e $\mathrm{Wu}$, com suporte pleno, já foram testadas anteriormente e os resultados colhidos foram comparados com soluções analíticas disponíveis, indicando aquelas com melhor desempenho. Os domínios, contudo, foram relativamente simples, apresentando formatos retangulares e circulares. Neste trabalho, mostra-se um exemplo de simulação da MECID com duas das melhores funções na solução de problemas geometricamente mais elaborados. A comparação dos resultados foi feita com o Método dos Elementos Finitos.
\end{abstract}

Palavras-chave. Problemas de Autovalores, Problemas de Helmholtz, Funcões de Base Radial no Método dos Elementos de Contorno.

\section{Introdução}

Atualmente, o emprego de funções escalares dependentes da distância euclidiana entre dois pontos, denominadas de Funções de Base Radial (FBR) substitui com vantagem os procedimentos de interpolação polinomiais em várias aplicações, particularmente quando se trata de aproximar dados esparsos em várias dimensões [3], mas também se mostram efetivas no ajuste de curvas e na solução de equações diferenciais. Grande parte do avanço no desenvolvimento de tais funções foi devido à aplicação delas na solução de problemas de geração e reestruturação de malhas em técnicas adaptativas, particularmente no contexto do Método dos Elementos Finitos (MEF). Para garantir que um ponto nodal não interaja com todos os demais pontos, mas apenas com os mais próximos. Neste sentido, certas funções radiais possuem um suporte compacto (FBRCs), em que acima de determinado valor do raio a função é nula [8]. Assim são formadas matrizes com diagonal dominante

\footnotetext{
${ }^{1}$ carlosloeffler@bol.com.br

2 engercules@gmail.com
} 
e positividade, além da simetria radial. O uso de FBRs no Método dos Elementos de Contorno principiou com o Método da Dupla Reciprocidade (MECDR) por [7], visando interpolar a variável que compõe o núcleo das integrais de domínio e servir como alternativa para a solução de problemas modelados por operadores não auto-adjuntos, além de usar uma solução fundamental mais simples. Contudo, há muitas restrições quanto ao desempenho da MECDR, relacionadas principalmente a forma como se processa a interpolação com as FBRs. Problemas ainda mais sérios, relacionados à instabilidade são encontrados na implementação de FBRCs com suporte compacto na MECDR, atenuadas apenas com a utilização de técnicas auxiliares [4]. Assim, as FBRCs devem ser usadas na MECDR com o suporte completo, embora sujeitas aos mesmos óbices das FBRs. Recentemente, foi desenvolvida uma técnica alternativa denominada Método dos Elementos de Contorno com Interpolação Direta (MECID), mais simples e similar ao um procedimento de interpolação, também usando primitivas das FBRs para transformar integrais do domínio em integrais de contorno. O MECID foi aplicado com sucesso em problemas de Poisson e de Helmhotz, utilizando FBRs clássicas [5]. O uso de FBRCs com suporte compacto, particularmente as funções propostas Wendlang e Wu, também foi feito em problemas de Poisson [5], mas os resultados não foram promissores. Verificou-se ainda que muitas das funções propostas, especialmente as de maior ordem, não tinham desempenho aceitável mesmo com suporte pleno. Tal como ocorreu na MECDR, uma pesquisa minuciosa para identificar o desempenho das principais FBRs e FBRCs deve ser realizada. Particularmente interessante é a simulação de problemas de autovalor, que permite uma avaliação bem precisa dos resultados, desde que se disponha de uma solução de referência, seja analítica ou obtida por um método numérico robusto, empregando uma malha bastante refinada. Já foram testadas na MECID, em problemas com domínios regulares, algumas FBRs tradicionais e outras, de Wendland, com suporte pleno. Estas últimas foram usadas não com o propósito de limitar seu raio de ação, mas sim por gerarem matrizes com diagonais dominantes, de onde resultaria maior precisão na solução do sistema final de equações discretas. O objetivo agora é simular problemas nos quais o domínio seja menos regular, oferecendo maior dificuldade numérica ao MECID e permitindo uma melhor avaliação da sua potencialidade. Apenas os resultados de duas funções radiais, identificadas em pesquisas anteriores como as de melhor desempenho, são aqui apresentados por questão de espaço.

\section{Formulação Integral}

Considere a equação de Helmholtz em sua forma integral inversa [2], dada pela equação (1):

$$
c(\xi) u(\xi)+\int_{\Gamma} u(X) q^{*}(\xi ; X) d \Gamma-\int_{\Gamma} q(X) u^{*}(\xi ; X) d \Gamma=\frac{1}{k^{2}} \omega^{2} \int_{\Omega} u(X) u^{*}(\xi ; X) d \Omega
$$

Na equação (1), $u(X)$ é o potencial escalar e $q(X)$ sua derivada normal; $u^{*}(\xi, X)$ é a solução fundamental e $\mathrm{q}^{*}(\xi, \mathrm{X})$ é sua derivada normal; $\omega$ é a freqüência de excitação associada. O coeficiente $\mathrm{c}(\xi)$ depende do posicionamento do ponto $\xi$ com relação ao domínio físico $\Omega(\mathrm{X})$. Na MECID, a solução fundamental também compõe o núcleo a ser 
interpolado e para que o ponto fonte $\xi$ possa ter a mesma posição dos pontos campo X, é preciso efetuar um procedimento de regularização, similar ao de Hadamard [1]. Assim sendo, faz-se (2):

$$
\begin{gathered}
c(\xi) u(\xi)+\int_{\Gamma} u(X) q^{*}(\xi ; X) d \Gamma-\int_{\Gamma} q(X) u^{*}(\xi ; X) d \Gamma= \\
\frac{1}{k^{2}} \omega^{2}\left\{\int_{\Omega}\left[u(X) u^{*}(\xi ; X)\right] d \Omega-\int_{\Omega}\left[u(\xi) u^{*}(\xi ; X)\right] d \Omega\right\}+\frac{1}{k^{2}} \omega^{2} \int_{\Omega}\left[u(\xi) u^{*}(\xi ; X)\right] d \Omega
\end{gathered}
$$

As duas primeiras integrais da equação (2) são aproximadas da seguinte forma:

$$
\frac{1}{k^{2}} \omega^{2}\left\{\int_{\Omega}\left[u(X) u^{*}(\xi ; X)\right] d \Omega-\int_{\Omega}\left[u(\xi) u^{*}(\xi ; X)\right] d \Omega\right\} \approx \frac{1}{k^{2}} \omega^{2}\left\{\int_{\Omega} \xi \alpha^{i} F^{i}\left(\mathbf{X}^{i} ; \mathbf{X}\right) d \Omega\right\}
$$

Na equação (3), $F^{i}$ representa a FBR adotada. Para cada ponto fonte $\xi$, a interpolação da MECID corresponde a uma varredura de todos os pontos base $X^{i}$ em relação aos pontos $\mathrm{X}$ do domínio, ponderada pelos coeficientes ${ }^{\xi} \alpha^{i}$. Uma função primitiva $\psi^{j}$ é utilizada para transformar a integral do domínio numa integral de contorno, ou seja:

$$
\int_{\Omega} \xi \alpha^{i} F^{i}\left(X^{i} ; X\right) d \Omega=\int_{\Omega}\left({ }^{\xi} \alpha^{j} \psi^{j}{ }_{, i i}(X)\right) d \Omega={ }^{\xi} \alpha^{j} \int_{\Omega} \psi_{, i}^{j}(X) n_{i}(X) d \Omega={ }^{\xi} \alpha^{j} \int_{\Gamma} \eta^{j}(X) d \Gamma
$$

Voltando-se à equação (2), o último termo do lado direito desta pode ser transformado numa integral de contorno, usando-se o conceito do Tensor de Galerkin $G^{*}$ [2]:

$$
\int_{\Omega}\left[u(\xi) u^{*}(\xi ; X)\right] d \Omega=u(\xi) \int_{\Omega}\left[G^{*}{ }_{, i i}(\xi ; X)\right] d \Omega=u(\xi) \int_{\Gamma}\left[G^{*}{ }_{, i}(\xi ; X) n_{i}(X)\right] d \Gamma
$$

Substituindo as equações (4) e (5) na equação (1) e utilizando-se dos procedimentos típicos de discretização do MEC, em que são considerados $\mathrm{n}$ pontos fonte, entre valores internos e pontos nodais de contorno, chega-se a seguinte equação matricial (6), cujo detalhamento pode ser colhido em [6]

$$
\left[\begin{array}{cc}
H_{11 . .} & H_{1 n} \\
. . & \\
H_{n 1} . . & H_{n n}
\end{array}\right]\left[\begin{array}{c}
\mathbf{u}_{1} \\
: \\
\mathbf{u}_{n}
\end{array}\right]-\left[\begin{array}{cc}
G_{11 . .} & G_{1 n} \\
. . & \\
G_{n 1} . . & G_{n n}
\end{array}\right]\left[\begin{array}{c}
\mathbf{q}_{1} \\
: \\
\mathbf{q}_{n}
\end{array}\right]=\frac{\omega^{2}}{k^{2}}\left[\begin{array}{cc}
{ }^{2} \alpha^{1} . . & { }^{1} \alpha^{m} \\
. . & \\
{ }^{2} \alpha^{1} . . & { }^{n} \alpha^{m}
\end{array}\right]\left[\begin{array}{c}
N_{1} \\
: \\
N_{m}
\end{array}\right]-\frac{\omega^{2}}{k^{2}}\left[\begin{array}{c}
Z_{1} \\
: \\
Z_{n}
\end{array}\right]
$$

Onde:

$$
\left[\begin{array}{c}
Z_{1} \\
: \\
Z_{n}
\end{array}\right]=\left[\begin{array}{cc}
{\left[\int_{1}{ }^{1} P^{1} d \Gamma_{1}+. .+\int_{n}{ }^{1} P^{n} d \Gamma_{n}\right] . .} & 0 \\
\ldots & {\left[\int_{1}{ }^{n} P^{1} d \Gamma_{1}+\ldots+\int_{n}{ }^{n} P^{n} d \Gamma_{n}\right]}
\end{array}\right]\left[\begin{array}{c}
\mathbf{u}_{1} \\
: \\
\mathbf{u}_{n}
\end{array}\right]
$$


Os coeficientes ${ }^{\xi} \alpha^{i}$ são as incógnitas, obtidas através da solução do seguinte sistema de equações algébricas, em que a solução fundamental figura numa matriz diagonal $\Lambda$ :

$$
\left.{ }^{\xi} \boldsymbol{\alpha}\right]=[\boldsymbol{F}]^{-1}\left[{ }^{\xi} \boldsymbol{\Lambda}\right][\boldsymbol{F}] \boldsymbol{\alpha}=[\boldsymbol{F}]^{-1}\left[{ }^{\xi} \boldsymbol{\Lambda}\right][\boldsymbol{u}]
$$

Para cada ponto fonte, demonstra-se que é possível alterar a ordem do produto matricial que aparece no lado direito da equação(7), explicitando o potencial u nos nós, de modo que:

$$
A_{\xi}=\left[\begin{array}{lll}
N_{1} & . . & N_{m}
\end{array}\right]\left[\begin{array}{c}
\xi \\
\alpha_{1} \\
: \\
\xi \alpha_{m}
\end{array}\right]
$$

Então, o valor de cada termo $A_{\xi}$ é calculado por:

$$
\left[\begin{array}{c}
A_{1} \\
: \\
A_{n}
\end{array}\right]=\left[\begin{array}{cc}
{\left[-S_{2}{ }^{1} \Lambda^{2} \ldots-S_{n}{ }^{1} \Lambda^{n}\right] . .} & S_{n}{ }^{1} \Lambda^{n} \\
. . & \\
S_{1}{ }^{n} \Lambda^{1} . . & {\left[-S_{1}{ }^{n} \Lambda^{1} \ldots-S_{n-1}{ }^{n} \Lambda^{n-1}\right]}
\end{array}\right]\left[\begin{array}{c}
\mathbf{u}_{1} \\
: \\
\mathbf{u}_{n}
\end{array}\right]
$$

O sistema matricial completo pode ser escrito como:

$$
\begin{aligned}
& {\left[\begin{array}{cc}
H_{11 . .} & H_{1 n} \\
. . & \\
H_{n 1 . .} & H_{n n}
\end{array}\right]\left[\begin{array}{c}
\mathbf{u}_{1} \\
: \\
\mathbf{u}_{n}
\end{array}\right]-\left[\begin{array}{cc}
G_{11 . .} & G_{1 n} \\
. . & \\
G_{n 1 . .} & G_{n n}
\end{array}\right]\left[\begin{array}{c}
\mathbf{q}_{1} \\
: \\
\mathbf{q}_{n}
\end{array}\right]=\frac{\omega^{2}}{k^{2}}\left[\begin{array}{c}
\left(A_{1}-Z_{1}\right) \\
: \\
\left(A_{n}-Z_{n}\right)
\end{array}\right]=} \\
& \frac{\omega^{2}}{k^{2}}\left[\begin{array}{cc}
M_{11 . .} & M_{1 n} \\
. . & \\
M_{n 1} . . & M_{n n}
\end{array}\right]\left[\begin{array}{c}
\mathbf{u}_{1} \\
: \\
\mathbf{u}_{n}
\end{array}\right]
\end{aligned}
$$

\section{Modelagem do Problema de Autovalor}

No MEC, o sistema matricial envolve simultaneamente os valores do potencial quanto de sua derivada normal. Assim, a equação (11) é manipulada para representar o problema de vibração livre. Usando-se submatrizes para destacar os valores nodais de u e q prescritos, tem-se:

$$
\left[\begin{array}{cc}
H_{u \bar{u}} & H_{u \bar{q}} \\
H_{q \bar{u}} & H_{q \bar{q}}
\end{array}\right]\left[\begin{array}{l}
\bar{u} \\
u
\end{array}\right]-\left[\begin{array}{ll}
G_{u \bar{u}} & G_{u \bar{q}} \\
G_{q \bar{u}} & G_{q \bar{q}}
\end{array}\right]\left[\begin{array}{c}
q \\
\bar{q}
\end{array}\right]=\frac{\omega^{2}}{k^{2}}\left[\begin{array}{ll}
M_{u \bar{u}} & M_{u \bar{q}} \\
M_{q \bar{u}} & M_{q \bar{q}}
\end{array}\right]\left[\begin{array}{c}
\bar{u} \\
u
\end{array}\right]
$$

Considerando que para este tipo de problema os valores nodais prescritos de $\mathrm{u}(\mathrm{X})$ e $\mathrm{q}(\mathrm{X})$ são nulos, eliminando-se a derivada do potencial q e condensando as diversas submatrizes da equação (12) encontra-se:

$$
[\bar{H}]\{\boldsymbol{u}\}=\frac{\omega^{2}}{\boldsymbol{k}^{2}}[\bar{M}]\{\boldsymbol{u}\}
$$




\section{Funções Radiais Utilizadas}

A Tabela (1) mostra as duas funções radiais escolhidas pelo bom desempenho mostrado anteriormente. Nesta, $\delta$ significa o valor do suporte e r é a distância entre os pontos base e campo. FBRCs de alta ordem, mesmo com suporte pleno, apresentaram problemas numéricos, provavelmente devido ao efeito da transformação de contorno representada na equação (4).

Tabela 1: Funções radiais.

\begin{tabular}{|c|c|c|}
\hline Nomeclatura & Funções de Base Radial & Primitivas associadas \\
\hline Spline Plate Function & $F^{i}(\mathrm{r})=\left(\frac{r}{\delta}\right)^{2} \ln \left(\frac{r}{\delta}\right)$ & $\psi_{, i}=\frac{1}{\delta^{2}}\left[-\frac{r^{2}}{16}+\frac{r^{2} \ln r}{4}\right] r_{i}$ \\
\hline Wendland & $F^{i}(\mathrm{r})=\left[1-\frac{r}{\delta}\right]_{+}^{3}$ & {$\left[\frac{1}{2}-\left(\frac{r}{\delta}\right)+\frac{3}{4}\left(\frac{r}{\delta}\right)^{2}-\frac{1}{5}\left(\frac{r}{\delta}\right)^{3}\right] r_{i}$} \\
\hline
\end{tabular}

\section{Exemplos de Simulação}

Dentre os casos resolvidos, escolheu-se um exemplo em que o domínio tem a forma de um X. As múltiplas reentrâncias do contorno oferecem uma boa oportunidade para avaliação da capacidade da MECID na solução de problemas não regulares, ao mesmo tempo em que pode se analisar o comportamento das duas funções radiais escolhidas. Usaram-se três diferentes malhas com elementos lineares e nós duplos em todas as quinas, conforme mostra a figura (1). A base do $\mathrm{X}$ foi fixada enquanto todos os demais contornos estão livres. A malha (a) possui 268 pontos nodais e 529 pontos internos interpolantes; a malha (b) possui os mesmos 268 pontos nodais com 817 pontos interpolantes; na terceira malha (c) tanto a quantidade de pontos nodais quanto pontos interpolantes foi aumentada, sendo 524 pontos nodais e 1043 pontos internos. Embora a MECID seja mais robusta do que a MECDR, as malhas da primeira necessitam de um número maior de pontos internos interpolantes. Daí a razão das malhas da MECID serem bastante densas de pontos no interior. Como não há solução analítica, foi aplicado o Método dos Elementos Finitos neste mesmo problema, afim de gerar resultados de referência. Utilizou-se para este caso elementos finitos isoparamétricos triangulares. A malha gerada pode ser observada em (d) na figura (1), nesta o refinamento consta com 2283 nós e 4308 elementos. Os resultados obtidos para os dez primeiros autovalores são apresentados na tabela (2), onde PC indicam os pontos no contorno e PI os pontos internos.

Destaca-se que, em se tratando de problemas dinâmicos, é necessária uma quantidade elevada de graus de liberdade para que seja obtido um espectro de frequências naturais com relativa precisão, ainda mais no caso da solução do MEF ser tomada como referência para o MECID. 
Tabela 2: Funções radiais.

\begin{tabular}{|c|c|c|c|c|c|c|}
\hline MEF & \multicolumn{6}{|c|}{ MEC } \\
\hline 2283 & 268 & 268 & 268 & 268 & 524 & 524 \\
\hline 2200 & 200 & & & & $\mathrm{P}$ & PC \\
\hline NOS & $\mathrm{PC}$ & $\mathrm{PC}$ & PC & & 1043 & 1043 \\
\hline 4308 & 529 PI & 529 PI & 817 PI & $817 \mathrm{PI}$ & & PI \\
\hline ELEM & $\mathrm{r} 2 \log (\mathrm{r})$ & Wendl & $\mathrm{r} 2 \log (\mathrm{r})$ & Wendl & $\mathrm{r} 2 \log (\mathrm{r})$ & Wendl \\
\hline 0.97947 & 0.98010 & 0.98256 & 0.97996 & 0.98257 & 0.97927 & 0.97901 \\
\hline 1.90209 & 1.90386 & 1.91312 & 1.90357 & 1.91325 & 1.89759 & 1.89915 \\
\hline 2.92591 & 2.92611 & 2.93870 & 2.92643 & 2.93882 & 2.92681 & 2.92827 \\
\hline 3.86515 & 3.87896 & 3.87243 & 3.87978 & 3.87382 & 3.85821 & 3.86769 \\
\hline 4.77319 & 4.75208 & 4.78959 & 4.74964 & 4.79155 & 4.77229 & 4.78108 \\
\hline 5.47034 & 5.43567 & 5.52986 & 5.42700 & 5.53217 & 5.45926 & 5.49014 \\
\hline 6.43626 & 6.30912 & 6.44564 & 6.19282 & 6.45378 & 6.43159 & 6.45645 \\
\hline 6.92130 & 6.78318 & 6.98346 & 6.58701 & 6.99536 & 6.90561 & 6.95834 \\
\hline 7.29091 & 6.84609 & 7.31970 & 6.66264 & 7.33461 & 7.28192 & 7.32056 \\
\hline 7.78364 & 7.00237 & 7.79998 & 6.90290 & 7.81159 & 7.76978 & 7.82681 \\
\hline
\end{tabular}

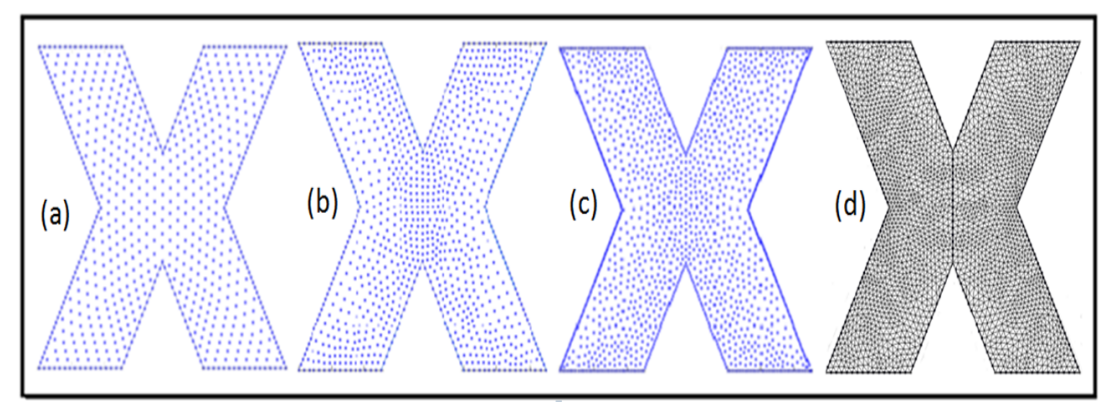

Figura 1: Três malhas de elementos de contorno lineares e uma de elementos finitos isoparamétricos utilizadas.

\section{Conclusão}

Pelos resultados obtidos, verifica-se que a MECID usando a FBRC de Wendland com suporte pleno resolveu satisfatoriamente o problema, mesmo para as malhas menos refinadas. No caso da FBR de placa fina, apenas a malha mais rica ofereceu bons resultados. Tal comportamento contrasta com o observado nas simulações já realizadas com domínios mais regulares, em que a FBR de placa fina retornou sempre resultados melhores do que quaisquer outras funções. A razão para tal comportamento, portanto, se deve ao fato da 
FBRC de Wendland gerar matrizes interpolantes com a diagonal dominante. Num exemplo com tais características geométricas, a questão do condicionamente matricial se torna muito importante, ressaltando que o sistema matricial do MEC não é simétrico. Contudo, o refinamento da malha de contorno e a inserção de maior quantidade de pontos internos interpolantes faz com que outras funções, não apenas a de placa fina, passe a responder satisfatoriamente. Embora não tenha sido mostrado, malhas muito pobres com a MECID - e neste caso a quantidade de pontos internos é igualmente importante - não conseguem gerar um espectro mais amplo de frequências; apenas com o refinamento da malha e a inserção de pontos no interior, calcula-se um número maior de frequências. Ressalta-se que a malha mais simples da MECID usando a FBRC retornou resultados melhores do que a malha menos refinada do MEF, particularmente no cálculo das últimas frequências. A similaridade dos resultados desta malha do MECID com a mais refinada do MEF foi bastante grande, mesmo usando uma quantidade de pontos nodais bem menor.

\section{Referências}

[1] C. L. R. Braga. Physical-Mathematics Notes (in portuguese). Editora Livraria da Física, São Paulo, 2006. ISBN 9798588325608

[2] C. A. Brebbia and J. Dominguez. Boundary Elements: An Introductory Course, WIT Press. 1992. ISBN: 978-1-85312-349-8

[3] M.D. Buhmann, Radial Basis Function: Theory and Implementations, Cambridge Press, 2003. ISBN 0521633389

[4] C. S. Chen, C. A. Brebbia and H. Power. Dual Reciprocity Method Using Compactly Supported Radial Basis Functions, Numerical Method in Biomedical Engineering, 15:137-150, 1999. DOI: 10.1002/(SICI)1099-0887(199902)15:2¡137::AIDCNM233¿3.0.CO;2-9.

[5] C. F. Loeffler, A. L. Cruz and A. Bulcão, A. Direct Use of Radial Basis Interpolation Functions for Modelling Source Terms with the Boundary Element Method. Engineering Analysis with Boundary Elements, 50:97-108, 2015. DOI: 10.1016/j.enganabound.2014.07.007

[6] C. F. Loeffler, L. Zamprogno, W. J. Mansur and A. Bulcão. Performance of Compact Radial Basis Functions in the Direct Interpolation Boundary Element Method for Solving Potential Problems.Computational Methods and Engineering and Sciences, 1:1-20, 2016. ISSN: 1526:1492

[7] P. W. Partridge, C. A. Brebbia and L.C. Wrobel. it The Dual Reciprocity Boundary Element Method, Computational Mechanics Publications and Elsevier, London, 1992, ISBN: 978-1-85166-700-0.

[8] H. Wendland. Piecewise polynomial, positive definite and compactly supported radial functions of minimal degree, Advances in Computational Mathematics, 4:389?-396, 1995. DOI: $10.1007 / \mathrm{BF} 02123482$. 